INRA Prod. Anim.,

2012,25 (2), $127-140$

\title{
Simplification des conduites d'élevage : analyse transversale des pratiques mises en ouvre dans les filières herbivores et granivores
}

\author{
N. HOSTIOU ${ }^{1,2,3,4}$, J. FAGON 5 \\ ${ }^{1}$ INRA, UMR1273 Métafort, F-63122 Saint-Genès-Champanelle, France \\ 2 AgroParisTech, UMR Métafort, BP 90054, F-63172, Aubière, France \\ ${ }^{3}$ Irstea, UMR Métafort, BP 50085, F-63172 Aubière, France \\ ${ }^{4}$ Clermont Université, VetAgro Sup, UMR Métafort, BP 35, F-63370 Lempdes, France \\ ${ }^{5}$ Institut de l'Elevage, Département Actions Régionales, Chemin de Borde Rouge, \\ F-31321 Castanet-Tolosan, France \\ Courriel :nhostiou@clermont.inra.fr
}

Les questions sur le travail sont depuis de nombreuses années au cœur des préoccupations des éleveurs et du monde professionnel. Les organismes de recherche-développement et les éleveurs mettent en œuvre des pratiques pour simplifier la conduite des troupeaux qui se différencient par leur nature, les conséquences sur le travail et sur l'équilibre du système d'élevage.

La question du travail est devenue incontournable pour qui s'intéresse aux mutations de l'élevage (Riedel et al 2007, Gallot 2008, Madelrieux et Dedieu 2008). En effet, les conditions de travail des éleveurs expliqueraient, en partie, la diminution du nombre d'exploitations et d'actifs agricoles, l'augmentation des cessations d'activité ainsi que le déficit d'installations (Rattin 2006). En France, depuis les années 1970, le nombre d'exploitations a fortement diminué, alors que les structures s'agrandissent (Chatellier et al 2008). L'agrandissement des élevages (Surface Agricole Utile (SAU) et cheptels) et la diminution de la maind'œuvre agricole induisent des charges en travail de plus en plus élevées pour les éleveurs (Caramelle-Holtz et al 2004). L'accroissement de la productivité du travail (4\% par an depuis 1979 pour la SAU/Unité Travailleur Année (UTA)) demeure une voie privilégiée de maintien de la compétitivité des exploitations (Rattin 2006, Sidot 2006). Le rapport des éleveurs à leur travail évolue également : les attentes d'un travail maîtrisé, distinct de la vie familiale, et la volonté de se préserver du temps libre prennent le pas sur le «labeur paysan» (Barthez 1986), où vie privée et travail sont confondus. Les éleveurs expriment de plus en plus la volonté d'avoir des horaires de travail maîtrisés, de partir en week-ends et en congés, autrement dit des horaires et des rythmes de travail qui ne les marginalisent pas vis-à-vis de leur famille et de la société (Guillaumin et al 2005). Pour diminuer la contrainte en travail, les éleveurs mobilisent conjointement ou indépendamment trois types de leviers : $i)$ simplifier la conduite technique du troupeau et/ou des surfaces, ii) recomposer la maind'œuvre, iii) améliorer les équipements et les bâtiments (Dedieu et Servière 2001). La simplification des pratiques d'élevage interpelle directement le champ de la zootechnie parce qu'elle a des conséquences sur les performances du troupeau et sur le fonctionnement du système d'élevage. En outre, ce volet reste encore peu étudié et analysé contrairement aux deux autres leviers. Les pratiques simplifiées sont très diverses par leur nature et leur contenu, car elles concernent les rythmes de traite, d'alimentation ou encore la reproduction qui sont les postes les plus chronophages en temps de travail dans les élevages herbivores et granivores (Chauvat et al 2003, Bartolomeu 2005, Gallot 2008). Les études sur les pratiques simplifiées se focalisent essentiellement sur une pratique particulière, en analysant les impacts sur les performances techniques et/ou économiques (Rémond et al 1999, Glesson et al 2008) et les conditions de leur insertion dans les élevages (Moreau et al 2004). Les pratiques simplifiées évoluent continuellement tant par les avancées de la recherche-développement que par les capacités d'innovation et d'adaptation des éleveurs ou encore les évolutions du contexte de production. De nouvelles pratiques se développent, tandis que d'autres tendent à disparaître. Dans le contexte actuel, la recherche de la maximisation de la production avec toujours moins de facteurs de production (exemple de l'augmentation de la productivité animale) rend difficile l'adoption de certaines pratiques simplifiées du fait des conséquences sur les performances $\mathrm{du}$ troupeau, et ce d'autant plus qu'il n'y a pas de compensation facile à mettre en œuvre, comme une augmentation des effectifs du troupeau. C'est par exemple le cas de la monotraite temporaire qui induit une diminution de la production laitière (Pomiès et al 2008). En outre, la notion de simplification des pratiques avec les troupeaux apparaît complexe et peut avoir plusieurs sens, nécessitant d'en préciser les contours. Elle semble recouvrir des significations différentes selon les productions 
animales où les enjeux de qualité de vie et de productivité du travail s'expriment différemment (Seegers et al 2006, Gallot 2008, Salaün 2008).

Les objectifs de cet article sont $: i$ ) de préciser les contours que recouvre la notion de «pratique simplifiée», ii) de faire un état des lieux des pratiques simplifiées dans les élevages herbivores (bovin, ovin, caprin) et granivores (porc, volaille) et iii) d'identifier les questions posées à la recherche-développement.

\section{1 / Que veut dire simplifier les pratiques?}

La notion de «simplification» des pratiques est souvent assimilée à la seule réduction de la durée du travail. Si «simplifier» («décharger quelqu'un de contraintes, de tâches fastidieuses, répétitives, prenantes») traduit bien l'idée de réduire le travail, une autre définition («rendre quelque chose plus simple, moins complexe, moins compliqué») traduit plutôt l'idée d'organiser différemment le travail (Larousse 2009). La modification de l'organisation et la réduction de la charge en travail, qui ressortent des enquêtes et de la bibliographie, se retrouvent bien dans ces deux définitions. Dans cet article, nous définissons une pratique simplifiée par le fait qu'elle renvoie à la réduction du nombre d'interventions nécessaires pour la réaliser durant l'année ou une période. Nous avons également pris en compte les pratiques, n'induisant pas une réduction du nombre d'actes techniques et donc du temps de travail, mais qui agissent sur les autres dimensions de la simplification évoquées auparavant (charge mentale, pénibilité physique...). En effet, les pratiques simplifiées répondent à des attentes diverses en termes de travail: réduire le temps de travail mais également diminuer la pénibilité du travail (physique et mentale), modifier la répartition des tâches dans l'année en évitant des moments difficiles à gérer ou encore sécuriser le fonctionnement du système d'élevage. Ces différents objectifs se retrouvent dans les attentes exprimées par les éleveurs sur leur travail, d'autant plus qu'une pratique est souvent reliée à une ou plusieurs attentes (Moreau et al 2004, Cournut et Hostiou 2010). Certaines pratiques simplifiées modifient le travail d'astreinte, c'est-à-dire le travail à réaliser quotidiennement (réduction du nombre de traites ou de distributions de la ration), alors que d'autres jouent sur le travail de saison (suppression de la récolte d'ensilage par exemple) (Bellet 2007). L'adoption d'une pratique simplifiée dans un élevage a parfois des répercussions plus globales que la seule réduction du temps de travail (modification des rythmes de traite avec des incidences sur le système fourrager par exemple) (Rubin et al 2006). L'étalement des mises bas réduit la charge de stress liée à la réussite de cette activité qui conditionne les résultats de la saison à venir, mais ne réduit pas le nombre d'actes et ne modifie pas le volume de travail à réaliser sur l'année (Kentzel 2007). En outre, les simplifications n'ont pas toutes les mêmes incidences sur le système d'élevage. Elles peuvent relever de l'ordre de l'ajustement de la conduite ou avoir des conséquences sur un ou plusieurs éléments du système, nécessitant parfois des compromis en termes d'attentes à satisfaire. En effet, lorsque les éleveurs intègrent de manière importante la question du travail dans leur projet, les équilibres et les performances techniques et/ou économiques du système d'élevage sont modifiés (Cournut et Hostiou 2010). C'est bien parce les attentes et les niveaux d'action sont divers que le terme de "simplification» renvoie à des notions plus complexes que la simple réduction du temps passé.

\section{2 / Enquête auprès d'ex- perts et analyse bibliogra- phique}

Une enquête a été réalisée auprès d'experts de terrain (Réseaux d'élevage, Institut de l'Elevage, IFIP, ITAVI et Chambres d'Agriculture) et de cher- cheurs investis dans les productions animales concernées (INRA, Irstea) (tableau 1). Le questionnaire a été réalisé lors d'un entretien avec l'expert ou renseigné directement par lui, suite à un envoi par courrier électronique. Les questions posées visaient à : $i$ ) définir les sens que ces experts donnent à l'expression «simplification du travail et adaptation des conduites d'élevage», ii) identifier les pratiques simplifiées actuellement en débat dans les élevages et étudiées par la recherche-développement, iii) qualifier leur dynamique d'évolution sur le terrain ainsi que les perspectives de recherche et de développement dans les années à venir. Pour compléter ces informations, la bibliographie scientifique et professionnelle a été mobilisée pour les différentes productions animales. La recherche bibliographique scientifique a été basée sur une requête agençant différents motsclés, choisis d'après le thésaurus AgroVoc proposé par la FAO. La requête a été réalisée sur la base de données «Web of Knowledge». Pour la bibliographie professionnelle, des recherches par mots clés sur les différentes pratiques simplifiées ont été réalisées dans des revues agricoles et des sites internet d'institutions agricoles (par exemple l'espace thématique Travail de l'Institut de l'Elevage ou encore sur ceux des Chambres d'Agriculture).

Un cadre d'analyse a été établi afin d'analyser les pratiques simplifiées selon : i) les productions animales concernées (bovin laitier, ovin laitier, caprin laitier, bovin allaitant, ovin allaitant, volailles, porcs), ii) leur contenu (nature et type), iii) les lieux des études réalisées (fermes expérimentales, fermes privées), iv) leur fréquence d'utilisation dans les fermes (faible, modérée, courante), v) leur dynamique d'adoption dans les fermes (recul, faible expansion, expansion), vi) les conséquences sur le travail (réduction du travail d'astreinte, répartition du travail sur l'année pour écrêter les pics de travail, atténuation de la charge physique...), vii) les conséquences sur le système d'élevage (effets sur les performances animales,

Tableau 1. Les experts enquêtés par filière et grande région d'élevage en France.

\begin{tabular}{|l|c|l|}
\hline Filière & $\begin{array}{c}\text { Nombre } \\
\text { d'experts }\end{array}$ & \multicolumn{1}{c|}{ Régions } \\
\hline Bovin lait & 3 & Midi-Pyrénées, Aquitaine, Bretagne \\
\hline Bovin viande & 3 & Pays de la Loire, Auvergne, Bourgogne, Aquitaine \\
\hline Ovin viande & 9 & Centre, Auvergne, Bourgogne, Midi-Pyrénées, Aquitaine \\
\hline Ovin lait & 2 & Aquitaine, Midi-Pyrénées, Corse \\
\hline Caprin lait & 4 & Rhône Alpes, PACA, Languedoc-Roussillon, Midi-Pyrénées, Aquitaine \\
\hline Volailles & 2 & France/Bretagne \\
\hline Porcs & 2 & Bretagne \\
\hline
\end{tabular}


Figure 1. Les pratiques simplifiées étudiées.

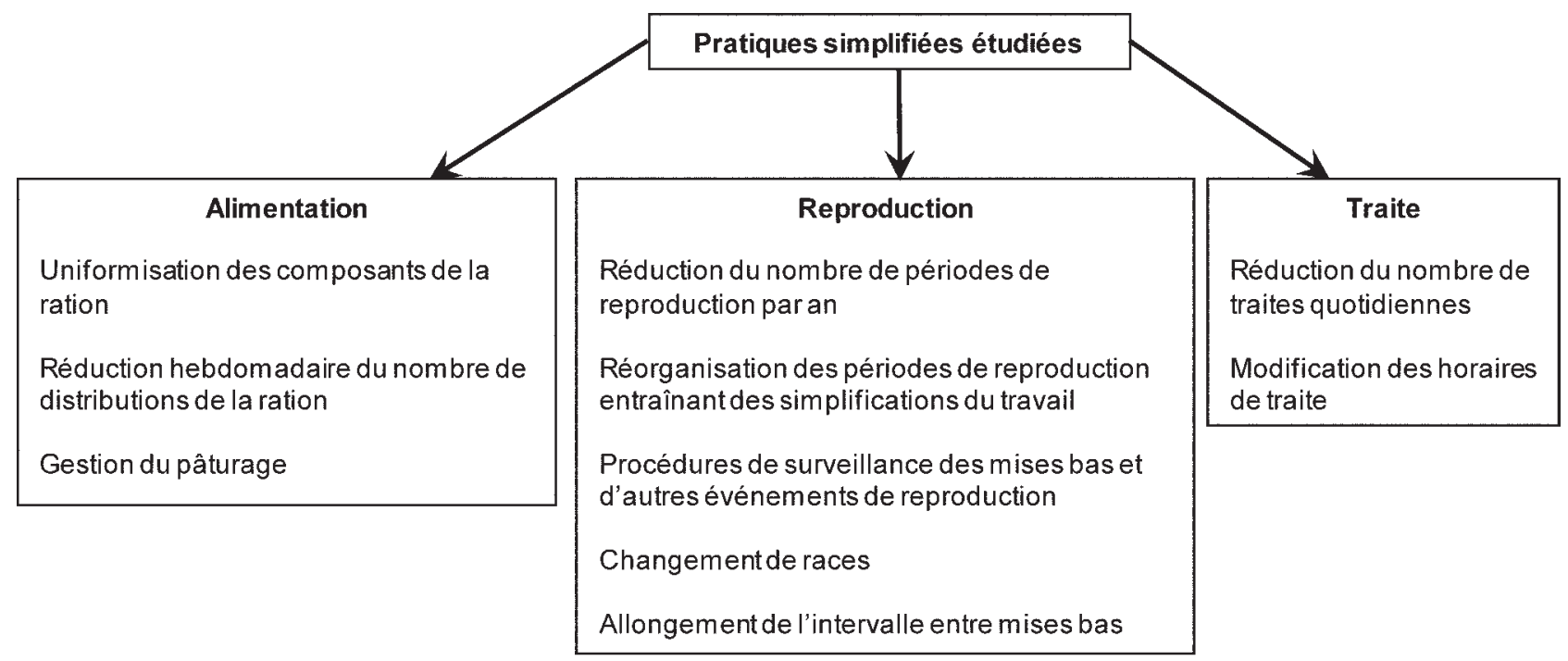

effets sur la conduite de l'élevage), ainsi que les liens aux deux autres leviers pour réorganiser le travail en élevage (main-d'œuvre, équipementbâtiment).

Ce cadre a été repris pour présenter cet article. Les pratiques simplifiées ont été regroupées selon trois grandes catégories constituant des postes particulièrement chronophages en temps de travail dans les exploitations : l'alimentation, la reproduction et la traite (figure 1). Les pratiques simplifiées pour l'alimentation et la reproduction sont présentées dans un premier temps car elles concernent l'ensemble des productions animales, au contraire de la traite.

\section{3 / Pratiques simplifiées pour l'alimentation}

Deux types de pratiques pour simplifier l'alimentation des animaux se sont développés au cours de ces dernières années: l'uniformisation des composants de la ration et la réduction hebdomadaire du nombre de distributions de la ration. Pour les herbivores, la gestion du pâturage fait également l'objet de pratiques simplifiées.

\section{1 / Uniformisation des compo- sants de la ration}

Le nombre de composants de la ration peut être réduit : à l'extrême, la ration est alors fondée sur l'apport d'un seul aliment qui est distribué à une ou plusieurs catégories d'animaux (exemple de la ration sèche, composée de concentrés et de foin ou paille, pour les jeunes animaux) (tableau 2), (Losq et Brunschwig 2009). Une deuxième pratique simplifiée repose sur la distribution d'une ration unique pour le troupeau, composée de nombreuses matières premières pour valoriser une gamme variée d'aliments, mais dont les temps de préparation et de distribution sont distincts, cette dernière tâche étant réalisée le plus souvent en une seule fois (ration complète pour les herbivores, soupe pour les porcs). La composition de la ration est raisonnée sur un objectif moyen pour chaque lot. Le recours à

Tableau 2. Analyse des pratiques simplifiées pour l'alimentation.

\begin{tabular}{|c|c|c|}
\hline Pratiques simplifiées & $\begin{array}{l}\text { Uniform isation des composants } \\
\text { de la ration }\end{array}$ & $\begin{array}{l}\text { Réduction hebdomadaire du nombre } \\
\text { de distributions de la ration }\end{array}$ \\
\hline Productions animales & Toutes & Herbivores \\
\hline Contenu & $\begin{array}{l}\text { Ration sèche, ration complète, } \\
\text { soupe (porcs) }\end{array}$ & $\begin{array}{l}\text { Adultes : distribution du fourrage en libre } \\
\text { service, } 1 \text { seule distribution/jour, } \\
4 \text { à } 6 \text { distributions/semaine } \\
\text { Jeunes: } 1 \text { buvée/jour, lait yogourt }\end{array}$ \\
\hline Lieux des études réalisées & $\begin{array}{l}\text { Fermes expérimentales, fermes } \\
\text { privées }\end{array}$ & Fermes expérimentales, fermes privées \\
\hline $\begin{array}{l}\text { Fréquence d'utilisation } \\
\text { dans les fermes }\end{array}$ & $\begin{array}{l}\text { Courante (ration complète et soupe) } \\
\text { ou faible (ration sèche) }\end{array}$ & Courante \\
\hline $\begin{array}{l}\text { Dynamique d'adoption } \\
\text { dans les fermes }\end{array}$ & Expansion & $\begin{array}{l}\text { Faible expansion ( } 2 \text { distributions/semaine } \\
\text { pour les jeunes) } \\
\text { Recul (libre service) }\end{array}$ \\
\hline Conséquences sur le travail & $\begin{array}{l}\text { Réduction du travail d'astreinte, } \\
\text { modification de la répartition } \\
\text { du travail sur l'année, réduction } \\
\text { de la pénibilité physique et mentale }\end{array}$ & $\begin{array}{l}\text { Réduction du travail d'astreinte, } \\
\text { modification de l'organisation du travail } \\
\text { pendant la journée }\end{array}$ \\
\hline $\begin{array}{l}\text { Conséquences } \\
\text { sur le système d'élevage }\end{array}$ & $\begin{array}{l}\text { Performances animales } \\
\text { (baisse de production) }\end{array}$ & $\begin{array}{l}\text { Ajustement de la conduite } \\
\text { Performances animales } \\
\text { (baisse de production) }\end{array}$ \\
\hline
\end{tabular}


une ration unique limite le nombre de distributions au cours de la journée avec un seul passage (Kentzel 2007). Ces pratiques concernent toutes les productions d'herbivores et de granivores. Elles semblent cependant plus avancées et répandues dans les exploitations porcines et avicoles où la gestion de l'alimentation se fait depuis longtemps par phase d'élevage (Bartolomeu 2005).

Ces deux pratiques simplifiées réduisent le nombre d'aliments à incorporer ainsi que le temps de préparation et de distribution des rations, et donc limitent le temps de travail d'astreinte (Caillaud 2006, Losq et Brunschwig 2009). Par exemple, la distribution de la ration complète évite l'apport de concentrés à distribuer en complément si cette tâche n'est pas automatisée. Si la ration sèche ne diminue pas le volume annuel de travail de saison, elle permet de le répartir différemment sur l'année. Sans ensilage de maïs, les chantiers qui lui sont associés (préparation du sol, semis, traitements...) sont supprimés comme celui très important de la récolte en fin d'été. Ils sont alors remplacés par des chantiers liés aux couverts végétaux implantés : récoltes d'herbe ou de céréales au printemps et en été (Caillaud 2006). La distribution d'une ration sèche identique toute l'année atténue la pénibilité du travail quotidien et évite le stress des transitions alimentaires surtout dans les systèmes spécialisés où le silo de maïs est ouvert toute l'année (Caillaud 2006). Elle est également un atout pour des éleveurs qui mettent en avant la prévisibilité et la sécurisation de leur système : la ration, basée sur des stocks, diminue la charge mentale par rapport à une alimentation basée sur le pâturage, dont le pilotage quotidien nécessite de nombreuses prises de décision.

Ces pratiques peuvent cependant altérer les performances économiques des exploitations (Losq et al 2009). La simplification du rationnement peut en effet avoir des effets sur les performances zootechniques selon la qualité du fourrage ou des concentrés apportés (appétence, encombrement et valeur nutritive). Ces pratiques n'ont pas la même fréquence d'utilisation dans les exploitations selon les productions animales. Ainsi, les rations complètes ou les soupes pour les porcs sont assez couramment mises en œuvre dans les élevages, contrairement aux rations sèches du fait de leur coût élevé. Ces pratiques simplifiées sont aussi souvent utilisées en complément avec les deux autres leviers («recomposition de la main-d'œuvre» et «équipement-bâtiment») pour réorganiser le travail: par exemple avec du matériel (bol ou remorque mélangeuse équipée d'un système de pesée pour la ration complète) ou avec des bâtiments aménagés pour optimiser le circuit de distribution ou déléguer la ration à une CUMA de distribution avec chauffeur.

\section{2 / Réduction hebdomadaire du nombre de distributions de la ration}

Une deuxième voie pour simplifier l'alimentation des animaux est de réduire le nombre de distributions hebdomadaires de la ration (tableau 2). Elle consiste à alimenter les animaux pour plusieurs jours, les concentrés complémentaires aux fourrages pour les herbivores pouvant être distribués quotidiennement ou avec la même fréquence. Elle concerne tous les élevages herbivores (Ferris et al 2006, Bastien et al 2009) mais pas les granivores, car l'alimentation fortement automatisée (machine à soupe, chaînes) induit moins de contraintes en temps de travail pour le poste «alimentation». Pour les animaux adultes ou le troupeau de renouvellement, les pratiques comprennent la distribution du fourrage en libre service, une seule distribution journalière ou encore quatre à six distributions hebdomadaires (Bastien et al 2009, Seite et al 2009). Pour les plus jeunes herbivores (ovin allaitant, bovin laitier et allaitant), les pratiques reposent sur la distribution du lait yogourt ${ }^{1}$, une seule buvée par jour, deux ou six distributions de lait par semaine (Brunschwig et Plouzin 2007).

Ces pratiques réduisent le travail d'astreinte ou modifient son organisation pendant la journée, voire la semaine, en réduisant le rythme de la tâche d'alimentation, en particulier lors de la période hivernale. Leur adoption induit une rupture avec des habitudes de travail structurant la journée, qui n'est pas évidente pour tous les éleveurs. Les deux voies (uniformiser les composants de la ration et réduire le nombre de distribution de la ration) peuvent se combiner entre elles et réduire de façon encore plus significative le temps passé à l'alimentation du troupeau. Par exemple, une ration complète peut être distribuée deux à trois fois par semaine ou une ration unique de foin peut être mise à disposition des animaux pour la semaine. L'adoption de ces pratiques simplifiées dans les élevages s'accompagne souvent d'un investissement dans du matériel de préparation et distribu- tion (bol mélangeur, mélangeuse-dessileuse-automotrice...).

La réduction du nombre de distributions hebdomadaires n'a pas de conséquence majeure sur le système d'élevage car il s'agit avant tout d'ajustements de la conduite. Mais ces pratiques peuvent avoir des conséquences sur les performances animales notamment à cause des problèmes de conservation de la qualité de la ration qui est distribuée pour plusieurs jours (Bastien et al 2009). Elles sont fréquemment adoptées par les éleveurs, mais elles ne sont pas toutes au même stade de développement. Par exemple, l'utilisation du libre service est en recul dans les exploitations laitières ovines et bovines. Les deux distributions par semaine pour les jeunes animaux restent encore au stade pionnier. Ces pratiques simplifiées sont étudiées par les organismes de développement dans des essais en stations expérimentales et par des analyses dans les élevages. Ainsi, de nombreuses études sont conduites en stations expérimentales pour mieux caractériser les impacts d'une réduction du nombre de distributions de la ration sur les performances animales comparativement à une distribution quotidienne (Bastien et al 2009).

\section{3 / Gestion du pâturage}

Plusieurs modes de conduite du pâturage existent et traduisent des organisations du travail différentes et des objectifs de production variables. Ces modes de conduites sont reliés aux contraintes des productions concernées ainsi qu'aux ressources disponibles, ou encore au système fourrager en place. Toutes les productions d'herbivores sont concernées même si la place du pâturage dans l'alimentation est très variable entre types de productions et entre régions. Les parcours enherbés concernent également les productions porcines et avicoles, mais ces dernières ne valorisent pas en tant que telle la ressource fourragère. L'aménagement des parcelles est logiquement moins étudié dans ces deux productions et se concentre sur l'occupation de l'espace et la circulation entre les cases pour les porcs en plein air par exemple. Dans les élevages d'herbivores, quatre principaux modes de conduite du pâturage ayant tous une incidence différente sur le travail et son organisation ont été recensés : le pâturage continu, tournant simplifié, tournant et rationné (figure 2). L'utilisation des surfaces en estive/alpage est proche d'un pâturage continu libre, mais nécessite un temps plus conséquent pour mener les animaux dans les parcelles et

\footnotetext{
${ }^{1}$ Le lait yogourt ou lait fermenté est un lait cru et froid, issu de l'ensemble du troupeau, qui fermente grâce à l'apport de bactéries lactiques (yaourt nature) pour obtenir un produit prédigéré destiné à l'alimentation des veaux et qui peut être distribué à tout moment de la journée, sans être réchauffé.
} 
Figure 2. Présentation de quatre modes de gestion du pâturage, et conséquences sur le temps de travail (Delaby communication personnelle). Les niveaux de gris représentent des parcelles de pâturage.

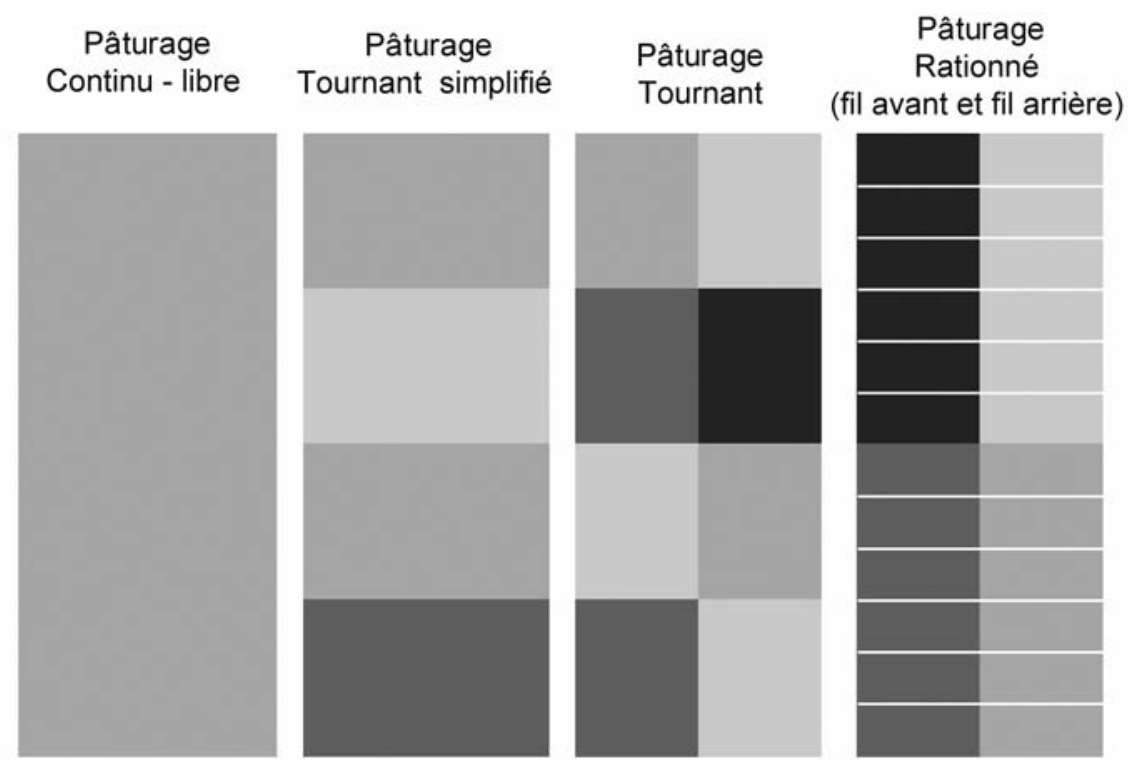

\section{- $\quad$ Temps de Travail}

(préparation des parcelles : clôture, chemin d'accès, approvisionnement en eau conduite : déplacement des fils avant et/ou arrière, déplacement des troupeaux)

du temps de surveillance car ils ne sont pas contenus dans un espace clos. Dans les quatre cas, les aménagements structurels à faire sur les parcelles avant leur exploitation par les animaux sont différents. Ainsi pour des pâtures conduites en continu, il y a très peu de clôtures intermédiaires à mettre en place (un talus ou des haies en délimitent parfois les contours), un seul point d'eau suffit (fonction de sa dimension) et un seul chemin d'accès à la parcelle est à prévoir. Le pilotage au quotidien est également très différent. Le déplacement des fils avant/fils arrière dans le cas du pâturage rationné est considéré comme très contraignant en termes de travail, même s'il permet de surveiller le troupeau. Bien souvent les logiques de production des éleveurs se retrouvent également derrière ces modes de conduite. Les éleveurs recherchant de bonnes performances animales souhaitent offrir autant que possible de l'herbe jeune et de bonne qualité à leur troupeau, ce qui se traduit par des rotations rapides sur les parcelles (pâturage rationné). Les pâturages tournant et tournant simplifié sont deux modes de conduite offrant un bon compromis entre le temps passé pour conduire les animaux sur les parcelles qui est moindre que pour du pâturage rationné, tout en préservant plus facilement la ressource qu'avec le pâturage continu (surface offerte aux animaux moins importante, donc moins de refus et de gaspillage) (Moreau et al 2004). Ils demandent du temps pour préparer les clôtures, mais des innovations techniques facilitent ce travail (pose de clôture en quad, élasticité des fils pour rouler dessus sans avoir à déposer les piquets). Les conditions pédo-climatiques et les systèmes fourragers en place dans les régions et les élevages permettent de tirer plus ou moins profit du pâturage. Plus la saison de pâturage est longue, plus les éleveurs économisent du temps de préparation et de distribution de fourrages complémentaires.

\section{4 / Pratiques simplifiées pour la reproduction}

\section{1 / Réduction du nombre de périodes de mise à la reproduc- tion par an}

Une pratique pour simplifier la reproduction est de réduire le nombre de périodes de reproduction par an et donc le nombre d'actes techniques liés à la gestion de la reproduction et des mises bas. Cette pratique est utilisée en élevage ovin allaitant et porcin (tableau 3). En ovin allaitant, cette pratique consiste à réduire le nombre de mises bas par brebis par exemple avec un agnelage par brebis et par an, tout en essayant de centrer cette période sur 1'herbe disponible (Benoit et Laignel 2009). En élevage porcin naisseur ou naisseurengraisseur, le rythme hebdomadaire ou pluri-hebdomadaire du travail est modi- fié avec l'adoption des conduites en 4 ou 5 bandes au lieu de la conduite en 7 bandes, plus couramment utilisée par les éleveurs. Ces pratiques simplifiées visent à organiser différemment le travail sur l'année, en particulier pour gérer des périodes plus creuses. Dans les exploitations porcines, la conduite en 4 ou 5 bandes (figure 3) permet aux éleveurs d'alterner des semaines avec moins de travail et de le différer sur d'autres pics de travail (Grannec 2010), ce que ne propose pas la conduite en 7 bandes, où chaque semaine, des tâches spécifiques sont à effectuer sur les truies (mise bas, sevrage ou insémination). Pour ce dernier mode de conduite, le travail est mieux réparti mais n'offre pas de semaines plus souples. Si ces pratiques restent encore peu mises en œuvre par les éleveurs, elles ont tendance à se développer. Elles ont un impact à l'échelle du système d'élevage car elles modifient les périodes de reproduction, la gestion de l'alimentation et le renouvellement du troupeau. Elles entraînent également une baisse de la productivité numérique annuelle par femelle en élevage ovin allaitant. En élevage porcin, elles induisent des risques sanitaires plus élevés du fait de la taille des bandes plus importante et d'un sevrage des porcelets à 21 jours au lieu de 28 jours.

\section{2 / Réorganisation des périodes de reproduction entraînant des simplifications du travail}

Des pratiques de réorganisation des périodes de reproduction n'induisent pas une réduction du nombre d'actes techniques mais entraînent d'autres formes de simplification du travail. Ces pratiques concernent le groupage des mises bas (Broccard et al 2005, Trou et al 2009, Grannec 2010) ou leur étalement sur l'année (Cournut et Dedieu 2005). Toutes les productions sont concernées sauf les ateliers d'engraissement (porcs, bovins), et la filière avicole qui a depuis longtemps segmenté et spécialisé les phases d'élevage (tableau 4). Les productions animales se différencient par la durée, le nombre de périodes de mises bas ainsi que le nombre de lots d'animaux à conduire au cours de l'année. Les besoins des filières ainsi que la physiologie de certaines espèces (ovine et caprine) structurent très fortement les stratégies de reproduction. Ainsi, en élevage ovin (lait et viande) et caprin, les agnelages ou les chevrotages peuvent être regroupés sur des périodes très courtes, de l'ordre de quelques semaines. La conduite de plusieurs lots de femelles permet alors de gérer plusieurs périodes de mises bas (Bossis 2006, Benoit et Laignel 2009). Dans le cas de lutte de contre-saison, le groupage est obtenu en utilisant la 
Tableau 3. Analyse des pratiques simplifiées réduisant le nombre d'interventions pour la reproduction.

\begin{tabular}{|c|c|c|c|}
\hline Pratiques simplifiées & $\begin{array}{c}\text { Réduction du nombre } \\
\text { de périodes } \\
\text { de reproduction par an }\end{array}$ & $\begin{array}{l}\text { Procédures de surveillance } \\
\text { des mises bas et d'autres } \\
\text { événements de reproduction }\end{array}$ & $\begin{array}{c}\text { Allongement } \\
\text { de l'intervalle } \\
\text { entre mises bas }\end{array}$ \\
\hline Productions animales & Ovin allaitant, porc & Toutes & Bovin lait, caprin lait \\
\hline Contenu & $\begin{array}{l}1 \text { agnelage/brebis/an } \\
\text { Conduite en } 4 \text { ou } 5 \text { bandes }\end{array}$ & $\begin{array}{l}\text { Suppression tournée du soir, } \\
\text { suppression des IA les } \\
\text { weekends ou allongement de } \\
\text { l'intervalle entre } 2 \text { IA (porc) }\end{array}$ & $\begin{array}{l}\text { Lactations longues ( }+ \text { de } \\
18 \text { mois entre } 2 \text { mises bas } \\
\text { en bovin laitier) }\end{array}$ \\
\hline $\begin{array}{l}\text { Lieux des études } \\
\text { réalisées }\end{array}$ & $\begin{array}{l}\text { Fermes expérimentales, } \\
\text { fermes privées }\end{array}$ & Fermes privees & Fermes expérimentales \\
\hline $\begin{array}{l}\text { Fréquence } \\
\text { d'utilisation } \\
\text { dans les fermes }\end{array}$ & Faible & $\begin{array}{l}\text { Modéré (volailles) } \\
\text { Modéré voire faible dans les } \\
\text { autres productions }\end{array}$ & Faible \\
\hline $\begin{array}{l}\text { Dynamique } \\
\text { d'adoption } \\
\text { dans les fermes }\end{array}$ & Faible & Faible & Faible \\
\hline $\begin{array}{l}\text { Conséquences } \\
\text { sur le travail }\end{array}$ & $\begin{array}{l}\text { Ovin allaitant: Modification } \\
\text { de la repartition du travail } \\
\text { d'astreinte pendant l'année } \\
\text { Porcs : Modification pluri } \\
\text { hebdomadaire du travail } \\
\text { (semaines avec moins de } \\
\text { travail alternant avec des } \\
\text { semaines avec plus de } \\
\text { travail) }\end{array}$ & Libérer du temps (soirées) & $\begin{array}{l}\text { Réduction de la fréquence } \\
\text { des inséminations et des } \\
\text { vêlages mais } \\
\text { augmentation du nombre } \\
\text { de jours de traite }\end{array}$ \\
\hline $\begin{array}{l}\text { Conséquences sur le } \\
\text { système d'élevage }\end{array}$ & $\begin{array}{l}\text { Modification des périodes } \\
\text { de reproduction, } \\
\text { Gestion de l'alimentation et } \\
\text { renouvellement }\end{array}$ & Ajustement de la conduite & $\begin{array}{l}\text { Modification des périodes } \\
\text { de reproduction }\end{array}$ \\
\hline
\end{tabular}

synchronisation par l'usage de traitements hormonaux. Les mises bas peuvent aussi être groupées sans être en lutte de contre-saison et en utilisant l'effet bélier. En bovin allaitant, les vêlages sont groupés sur une ou deux périodes de trois à quatre mois (Kentzel 2007). En bovin laitier, le groupage des mises bas se fait sur une période unique de trois à cinq mois (Trou et al 2009). Outre les performances de reproduction des animaux, le choix des périodes par les éleveurs résulte de la combinaison de plusieurs facteurs : leurs objectifs, les conditions pédo-climatiques et la production associée de fourrages (Cournut et Dedieu 2005) ainsi que les performances de reproduction des animaux. La filière joue également un rôle clé sur les choix des éleveurs du fait des périodes de production, en particulier par exemple dans les filières AOP pro- ductrices de fromages de brebis, ou de l'évolution des prix au cours de l'année.

En bovin laitier et allaitant, l'étalement des mises bas qui consiste à les répartir de manière relativement homogène tout au long de l'année est fondé sur une conduite à l'animal plutôt que sur une conduite en lot. Un fort étalement des mises bas amène certains élevages ovins allaitants à se rapprocher

Tableau 4. Analyse des pratiques entraînant une simplification du travail pour la reproduction.

\begin{tabular}{|c|c|c|}
\hline Pratiques simplifiées & $\begin{array}{c}\text { Réorganisation des périodes } \\
\text { de reproduction }\end{array}$ & Changement de races \\
\hline Productions animales & $\begin{array}{l}\text { Toutes (sauf engraissement en porcs } \\
\text { et en bovins, et sauf volailles) }\end{array}$ & Ovin allaitant, volailles \\
\hline Contenu & $\begin{array}{l}\text { Groupage ou etalement des mises } \\
\text { bas }\end{array}$ & Races plus rustiques \\
\hline Lieux des études réalisées & $\begin{array}{l}\text { Fermes experimentales, fermes } \\
\text { privées }\end{array}$ & $\begin{array}{l}\text { Fermes expérimentales, fermes } \\
\text { privées }\end{array}$ \\
\hline $\begin{array}{l}\text { Fréquence d'utilisation } \\
\text { dans les fermes }\end{array}$ & Modérée & Faible \\
\hline $\begin{array}{l}\text { Dynamique d'adoption } \\
\text { dans les fermes }\end{array}$ & En expansion & $\begin{array}{l}\text { Faible } \\
\text { En expansion (dindes) }\end{array}$ \\
\hline Conséquences sur le travail & $\begin{array}{l}\text { Modification de l'organisation du } \\
\text { travail sur l'annee (répartition, charge } \\
\text { mentale, temps libre) }\end{array}$ & $\begin{array}{l}\text { Réduction du travail d'astreinte, } \\
\text { madification de la répartition du } \\
\text { travail sur l'année }\end{array}$ \\
\hline $\begin{array}{l}\text { Conséquences sur le système } \\
\text { d'élevage }\end{array}$ & $\begin{array}{l}\text { Modification de la conduite } \\
\text { (allotement, affourragement...) }\end{array}$ & $\begin{array}{l}\text { Modification de la conduite } \\
\text { (allotement, affourragement...) }\end{array}$ \\
\hline
\end{tabular}


Figure 3. Trois modes de conduite pour un atelier naisseur porcin, à partir de l'exemple d'un élevage avec 280 truies productives conduites en quatre, cinq ou plus classiquement en sept bandes.

Conduite en 4 Bandes : sevrage à 21 jours

\begin{tabular}{|c|c|c|c|c|c|c|c|c|c|c|c|c|c|c|c|c|c|c|c|c|c|c|}
\hline Truies/Bande & Semaines & 1 & 2 & 3 & 4 & 5 & 6 & 7 & 8 & 9 & 10 & 11 & 12 & 13 & 14 & 15 & 16 & 17 & 18 & 19 & 20 & 21 \\
\hline 70 & Bande 1 & $\begin{array}{l}M \\
B\end{array}$ & & & $\begin{array}{l}\mathrm{S} \\
\mathrm{e}\end{array}$ & $\begin{array}{l}S \\
i\end{array}$ & & & & & & & & & & & & & & & & $\begin{array}{l}M \\
B\end{array}$ \\
\hline 70 & Bande 2 & & & & & & $\begin{array}{l}M \\
B\end{array}$ & & & $\begin{array}{l}\mathrm{S} \\
\mathrm{e}\end{array}$ & $\begin{array}{l}\mathrm{S} \\
\mathrm{i}\end{array}$ & & & & & & & & & & & \\
\hline 70 & Bande 3 & & & & & & & & & & & $\begin{array}{l}M \\
B\end{array}$ & & & $\begin{array}{l}\mathrm{S} \\
\mathrm{e}\end{array}$ & $\begin{array}{l}\mathrm{S} \\
\mathrm{i}\end{array}$ & & & & & & \\
\hline 70 & Bande 4 & & & & & & & & & & & & & & & & $\begin{array}{l}M \\
B\end{array}$ & & & $\begin{array}{l}\mathrm{S} \\
\mathrm{e}\end{array}$ & $\begin{array}{l}\mathrm{S} \\
\mathrm{i}\end{array}$ & \\
\hline
\end{tabular}

Conduite en 5 Bandes : sevrage à 21 jours

\begin{tabular}{|c|c|c|c|c|c|c|c|c|c|c|c|c|c|c|c|c|c|c|c|c|c|c|}
\hline Truies/Bande & Semaines & 1 & 2 & 3 & 4 & 5 & 6 & 7 & 8 & 9 & 10 & 11 & 12 & 13 & 14 & 15 & 16 & 17 & 18 & 19 & 20 & 21 \\
\hline 56 & Bande 1 & $\begin{array}{l}\mathrm{M} \\
\mathrm{B}\end{array}$ & & & $\begin{array}{l}\mathrm{S} \\
\mathrm{e}\end{array}$ & $\begin{array}{l}S \\
i\end{array}$ & & & & & & & & & & & & & & & & $\begin{array}{l}\mathrm{M} \\
\mathrm{B}\end{array}$ \\
\hline 56 & Bande 2 & & & & & $\begin{array}{l}\text { M } \\
B\end{array}$ & & & $\begin{array}{l}S \\
\mathrm{e}\end{array}$ & $\begin{array}{l}\text { S } \\
\text { S }\end{array}$ & & & & & & & & & & & & \\
\hline 56 & Bande 3 & & & & & & & & & $\begin{array}{l}M \\
B\end{array}$ & & & $\begin{array}{l}\mathrm{S} \\
\mathrm{e}\end{array}$ & $\begin{array}{l}S \\
i\end{array}$ & & & & & & & & \\
\hline 56 & Bande 4 & & & & & & & & & & & & & $\begin{array}{l}M \\
B\end{array}$ & & & $\begin{array}{l}\text { S } \\
\text { e }\end{array}$ & $\begin{array}{l}S \\
i\end{array}$ & & & & \\
\hline 56 & Bande 5 & & & & & & & & & & & & & & & & & $\begin{array}{l}\mathrm{M} \\
\mathrm{B}\end{array}$ & & & $\begin{array}{l}\mathrm{S} \\
\mathrm{e}\end{array}$ & $\begin{array}{l}S \\
i\end{array}$ \\
\hline
\end{tabular}

Conduite en 7 Bandes : sevrage à 28 jours

\begin{tabular}{|c|c|c|c|c|c|c|c|c|c|c|c|c|c|c|c|c|c|c|c|c|c|c|}
\hline Truies/Bande & Semaines & 1 & 2 & 3 & 4 & 5 & 6 & 7 & 8 & 9 & 10 & 11 & 12 & 13 & 14 & 15 & 16 & 17 & 18 & 19 & 20 & 21 \\
\hline 40 & Bande 1 & $\begin{array}{l}M \\
B\end{array}$ & & & & $\begin{array}{l}S \\
\mathrm{e}\end{array}$ & $\begin{array}{l}\mathrm{S} \\
\mathrm{i}\end{array}$ & & & & & & & & & & & & & & & \\
\hline 40 & Bande 2 & & & & $\begin{array}{l}M \\
B\end{array}$ & & & & $\begin{array}{l}\text { S } \\
\text { e }\end{array}$ & $\begin{array}{l}\mathrm{S} \\
\mathrm{i}\end{array}$ & & & & & & & & & & & & \\
\hline 40 & Bande 3 & & & & & & & $\begin{array}{l}M \\
B\end{array}$ & & & & $\begin{array}{l}S \\
e\end{array}$ & $\begin{array}{l}\mathrm{S} \\
\mathrm{i}\end{array}$ & & & & & & & & & \\
\hline 40 & Bande 4 & & & & & & & & & & $\begin{array}{l}\text { M } \\
\text { B }\end{array}$ & & & & $\begin{array}{l}\mathrm{S} \\
\mathrm{e}\end{array}$ & $\begin{array}{l}\mathrm{S} \\
\mathrm{i}\end{array}$ & & & & & & \\
\hline 40 & Bande 5 & & & & & & & & & & & & & $\begin{array}{l}M \\
B\end{array}$ & & & & $\begin{array}{l}\mathrm{S} \\
\mathrm{e}\end{array}$ & $\begin{array}{l}S \\
i\end{array}$ & & & \\
\hline 40 & Bande 6 & & & & & & & & & & & & & & & & $\begin{array}{l}\text { M } \\
B\end{array}$ & & & & $\begin{array}{l}\mathrm{S} \\
\mathrm{e}\end{array}$ & $\begin{array}{l}\mathrm{S} \\
\mathrm{i}\end{array}$ \\
\hline 40 & Bande 7 & & $\begin{array}{l}\mathrm{S} \\
\mathrm{e}\end{array}$ & $\begin{array}{l}\mathrm{S} \\
\mathrm{i}\end{array}$ & & & & & & & & & & & & & & & & $\begin{array}{l}M \\
B\end{array}$ & & \\
\hline
\end{tabular}

MB Semaine Mises Bas

Se Semaine Sevrage
Semaine d'Allaitement

Si Semaine Insémination

Période de Gestation

d'une conduite en bande typique d'ateliers granivores, avec par exemple un lot d'agnelage par mois de septembre à juin (Bellet et al 2010). En élevage caprin et ovin laitier, l'étalement se fonde sur l'obtention de plusieurs périodes de mises bas par la conduite de deux ou trois lots de femelles (Morin 2003, Bossis 2006). Pour les ovins laitiers, il s'agit d'élevages pratiquant un début de traite décalé par rapport aux périodes de traite traditionnelle. Dans la plupart des élevages, les brebis sont conduites en un seul troupeau avec un ou plusieurs lots de lutte d'adultes et un ou plusieurs lots de lutte d'agnelles, avec un décalage de un à trois mois entre les adultes et les agnelles. Pour d'autres élevages, la conduite des brebis se fait en deux troupeaux avec un chevauchement des périodes de traite, par exemple, un troupeau avec des mises bas précoces, visant une production de contre-saison et un troupeau dont la production sera principalement livrée aux industriels de Roquefort (Morin et De Boissieu 2011). Le décalage des périodes de reproduction est avant tout lié à des demandes des filières laitières.

Ces pratiques ne réduisent pas la durée du travail d'astreinte mais permettent de l'organiser différemment (Trou et al 2009). Avec des mises bas groupées, le travail est rationalisé et organisé en séquences de tâches (alimentation, surveillance des mises bas) grâce à la gestion d'un lot (cas des bovins et des ovins laitiers) ou de plusieurs lots homogènes (cas des bovins et des ovins allaitants, et des caprins laitiers). Le travail d'astreinte et la charge mentale sont également allégés quand se terminent les périodes de mises bas. Les éleveurs se libèrent du temps pour gérer les pointes de travail, par exemple avec les cultures au printemps (Huchon et al 2006). Les mises bas étalées induisent une répartition homogène de la charge de travail sur l'année, permettant alors d'écrêter les pointes de travail avec le troupeau (Bossis 2006), et d'obtenir des entrées de trésorerie régulières. Cependant en élevage ovin laitier, l'étalement saisonnier des mises bas est parfois obtenu par la conduite de deux lots homogènes accroissant la charge en travail (alimentation, traite...). La réorganisation des périodes de reproduction sur l'année a des conséquences sur le système d'élevage en modifiant les équilibres entre travail, performances économiques et techniques. Par exemple, en bovin allaitant, les vaches sont conduites en lots 
homogènes modifiant ainsi l'affouragement et les périodes de vente des produits (Kentzel 2007). Ces pratiques sont fréquemment utilisées par les éleveurs. De nombreuses études sont réalisées sur ce thème en stations expérimentales pour mieux identifier les impacts techniques et économiques (Broccard et al 2007). Cependant dans certaines productions animales, les groupages ne peuvent pas se faire sans concessions sur les performances animales. Par exemple, pour maintenir des vêlages groupés en élevage bovin laitier, des vaches avec une bonne production laitière peuvent être réformées du fait de difficultés à être inséminées lors de la période adéquate. L'introduction de taureaux dans les élevages pour rattraper ces échecs est de plus en plus fréquente permettant ainsi de supprimer la tâche de détection des chaleurs par l'éleveur. Le groupage des vêlages est fréquemment associé à une alimentation herbagère, à la monotraite en fin de lactation des vaches laitières, voire à la fermeture de la salle de traite pendant quelques semaines en été ou en hiver (Ferris et al 2008, Cournut et Hostiou 2010). Cependant, dans le cadre de la réforme de la gestion des droits à produire, certaines laiteries souhaitent contractualiser avec les éleveurs en intégrant un critère sur des livraisons stables toute l'année, ce qui conduit à étaler les vêlages plutôt qu'à les grouper, pouvant remettre en cause l'utilisation de cette pratique.

\section{3 / Procédures de surveillance des mises bas et d'autres événe- ments de reproduction}

Au-delà de la conduite de la reproduction, un deuxième niveau de simplification possible a trait aux procédures de surveillance des mises bas et d'autres événements de reproduction (insémination artificielle par exemple). Des éleveurs ovins ou bovins retardent ou suppriment leur tournée du soir (tableau 3). Dans les élevages porcins avec un atelier naisseur, les éleveurs peuvent supprimer les inséminations les week-ends, ou allonger l'intervalle entre deux inséminations successives (Martel et al 2008). La filière avicole a depuis longtemps segmenté et spécialisé les phases d'élevage (sélection, multiplication et production), les enjeux autour de cette phase de reproduction concernant uniquement les deux premières catégories, qui représentent un nombre limité d'exploitations. Ces pratiques sont plus de l'ordre de l'ajustement car elles ne remettent pas en cause la conduite du troupeau et des surfaces. En revanche, elles reflètent bien la notion de compromis qui est en jeu lorsque l'on parle de simplification: compromis entre performances techniques, optimisation économique et incidences sur le travail. Ces dernières sont ponctuelles et permettent aux éleveurs de se libérer du temps à certains moments (par exemple une soirée ou un week-end) ou de gérer l'emploi du temps d'un salarié (par exemple en ne le faisant pas travailler la nuit). Des éleveurs vont associer à ces pratiques simplifiées des systèmes de vidéo-surveillance ou de nouveaux équipements pour faciliter la surveillance du troupeau (podomètre, bolus sonde pour enregistrer et transmettre des informations traduisant des modifications de l'activité des animaux telles que des troubles métaboliques, venue en chaleurs, imminence du vêlage) et limiter leur passage dans les bâtiments d'élevage, illustrant alors les possibilités d'associer plusieurs leviers (simplifier et s'équiper).

\section{4 / Changement de races et sélection génétique}

Des changements de races sont également considérés comme des pratiques simplifiant le travail de l'éleveur (tableau 4). En filière dinde de chair, l'utilisation progressive depuis plus de 3 ans de nouvelles souches génétiques (Grademaker, T10...), diminue les troubles digestifs des animaux et limite ainsi les problèmes de litière. Les éleveurs réduisent le nombre d'intervention, le temps passé pour contrôler l'ambiance dans les bâtiments, la fréquence de paillage et les pertes économiques. En ovin allaitant, afin de mieux répartir le travail sur l'année et d'approvisionner régulièrement la filière, des élevages herbagers développent des luttes en contre-saison (agnelages en bergerie). Pour cela, les éleveurs adoptent des races naturellement dessaisonnées qui ont des caractéristiques plus maternelles et dont l'agneau est traditionnellement élevé en bergerie (exemple de la Romane). L'adoption de ces races plus maternelles réduit le temps consacré à la surveillance lors des agnelages. D'après les experts enquêtés, la fréquence d'utilisation de cette pratique dans les exploitations est stable ou en expansion modérée. Des travaux sont en cours pour étudier si certaines races bovines sont plus adaptées que d'autres à un contexte de production, à un système d'élevage (bonne valorisation des ressources fourragères, moindre complémentation alimentaire) et pour observer les performances techniques (production, reproduction, santé) et les incidences économiques de ces différentes races (au Teagasc à Moorepark en Irlande, à l'INRA au Pin au Haras en Normandie) (Delaby et al 2009). L'impact sur la reproduction (Cutullic et al 2010) et les réponses aux différents régimes alimentaires pourraient apporter des éléments de conduite pour simplifier le travail des éleveurs.

\section{5 / Allongement de l'intervalle entre mises bas}

Pour simplifier 1'organisation des périodes de reproduction, une pratique consiste à allonger la durée entre mises bas. Elle est utilisée en élevage laitier bovin et caprin (tableau 3 ). Elle repose sur l'allongement de la durée de la lactation, appelée plus couramment «lactations longues») induisant une réduction du nombre d'actes techniques pour la gestion de la reproduction (inséminations, mises bas...) mais augmentant le nombre de jours de traite (Rotz et al 2005, Caramelle-Holtz et al 2008). Ainsi en bovin laitier, la mise à la reproduction peut avoir lieu tous les 18 mois (Porhiel 2008) si les animaux laitiers ont des niveaux de production élevés et persistants. Mais le travail n'est pas forcément au cœur des attentes des éleveurs car les lactations longues sont plus souvent présentées comme un moyen de pallier des problèmes de reproduction (Porhiel 2008). Les impacts économiques sont peu évalués, cette pratique étant encore peu utilisée et analysée. Des études en stations expérimentales visent à mieux les caractériser. Par exemple, un essai a été mené pendant trois ans (2005-2008) à la ferme expérimentale de Trévarez, en Bretagne. D'autres études en Europe ou dans le monde ont surtout été réalisées dans le cas d'allongements de lactation subis suite à des échecs répétés des inséminations des vaches (Rotz et al 2005, Auldist et al 2007, Sorensen et al 2008).

\section{5 / Pratiques simplifiées pour la traite}

Dans les exploitations laitières, la traite biquotidienne représente la principale charge de travail avec $50 \%$ de l'astreinte en bovin et $36 \%$ en caprin (Chauvat et al 2003, Caramelle-Holtz et al 2004) et a donc conduit à la recherche de pratiques simplifiées telles que la réduction du nombre de traites quotidiennes ou la modification des horaires de traite.

\section{1 / Réduction du nombre de traites quotidiennes}

La réduction de la fréquence de traite repose sur la monotraite annuelle ou saisonnière (une seule traite par jour à intervalle de $24 \mathrm{~h}$ ), ou l'arrêt de la traite avec la fermeture saisonnière de la salle de traite (Clark et al 2006, Armstrong et Ho 2007, Komara et al 2009) (tableau 5). Ces pratiques sont utilisées 
Tableau 5. Analyse des pratiques simplifiées pour la traite.

\begin{tabular}{|l|l|l|}
\hline Pratiques simplifiées & \multicolumn{1}{|c|}{$\begin{array}{c}\text { Réduction du nombre de traites } \\
\text { quotidiennes }\end{array}$} & Modification des horaires de traite \\
\hline Productions animales & Bovin, ovin, caprin & Bovin, caprin \\
\hline Contenu & $\begin{array}{l}\text { Monotraite annuelle et saisonnière, } \\
\text { fermeture saisonnière de la salle } \\
\text { de traite }\end{array}$ & $\begin{array}{l}\text { Suppression d'une traite } \\
\text { hebdomadaire, 3 traites en 2 jours, } \\
2 \text { traites à horaires rapprochés }\end{array}$ \\
\hline Lieux des études réalisées & $\begin{array}{l}\text { Fermes expérimentales, fermes } \\
\text { privées }\end{array}$ & $\begin{array}{l}\text { Fermes expérimentales, fermes } \\
\text { privées (suppression d'une traite } \\
\text { hebdomadaire) }\end{array}$ \\
\hline $\begin{array}{l}\text { Fréquence d'utilisation } \\
\text { dans les fermes }\end{array}$ & $\begin{array}{l}\text { Faible en élevage caprin et ovin } \\
\text { Modérée en bovin }\end{array}$ & $\begin{array}{l}\text { Important (suppression d'une traite } \\
\text { hebdomadaire) } \\
\text { Nulle pour les 2 traites à horaires } \\
\text { rapprochés }\end{array}$ \\
\hline $\begin{array}{l}\text { Dynamique d'adoption } \\
\text { dans les fermes }\end{array}$ & $\begin{array}{l}\text { Expansion (suppression d'une traite } \\
\text { hebdomadaire) } \\
\text { Nulle pour les 2 traites à horaires } \\
\text { rapprochés }\end{array}$ \\
\hline Conséquences sur le travail & $\begin{array}{l}\text { Réduction du travail d'astreinte, } \\
\text { modification de la répartition } \\
\text { du travail sur l'année }\end{array}$ & $\begin{array}{l}\text { Réduction ponctuelle du travail } \\
\text { d'astreinte, modification de la } \\
\text { répartition du travail sur la journée }\end{array}$ \\
\hline $\begin{array}{l}\text { Codification de la conduite } \\
\text { (reproduction, alimentation) } \\
\text { d'élevagéne des performances laitières } \\
\text { Augmentation de l'effectif } \\
\text { de femelles }\end{array}$ & $\begin{array}{l}\text { Ajustement affouragement } \\
\text { et complémentation }\end{array}$ \\
\hline
\end{tabular}

dans toutes les productions d'herbivores laitiers (bovin, ovin, caprin). Les exploitations laitières de petits ruminants, avec une gestion par lots, des mises bas très groupées, et des caractéristiques physiologiques (durée de lactation et persistance) ne permettant pas de produire sur une année entière, sont davantage concernées par les fermetures saisonnières de salle de traite que les bovins.

La suppression d'une traite journalière réduit le travail de une à deux heures par jour. Les pratiques simplifiées saisonnières (monotraite ou fermeture de la salle de traite au printemps et/ou en été) modifient également la répartition annuelle du travail d'astreinte. Il est également rendu plus flexible, en particulier lors de périodes où les interactions avec les autres tâches agricoles (récoltes de foin par exemple) ou des loisirs posent des problèmes de concurrence (Cournut et Dedieu 2005). Si ces pratiques s'avèrent très efficaces pour réduire le travail d'astreinte, leur mise en œuvre est toutefois limitée par la difficulté qu'ont les éleveurs à rompre avec un mode d'organisation séculaire (poids de l'histoire, du regard de la famille et des éleveurs voisins) mais aussi par les effets sur les performances laitières. Les éleveurs caprins seraient moins réticents à mettre en œuvre cette pratique sans craindre un trop fort impact de cet effet négatif du fait des baisses de performances laitières plus faibles par rapport aux vaches laitières (Davis et al 1999, Komara et Marnet 2009). Ces simplifications de la traite ont des conséquences sur le système d'élevage. Leur adoption amène souvent les éleveurs à concentrer les mises bas sur une courte période. La monotraite annuelle s'accompagne de stratégies de compensation de la baisse de la production par l'augmentation de l'effectif de femelles en production, et éventuellement des quantités de fourrages et de la nature des concentrés distribués. La réduction des rythmes de traite ne s'accompagne pas nécessairement d'une dégradation des performances économiques des élevages (Pomiès et al 2008). La sélection animale prend de plus en plus en compte ces critères de persistance ou de capacité à s'adapter à ces modifications des rythmes de traite.

Dans les élevages, ces pratiques sont utilisées en association avec d'autres simplifications (gestion par lots pour faciliter la reproduction et disposer ainsi de lots avec un stade de lactation plus homogène, alimentation collective comme la ration complète...) (Cournut et Hostiou 2010). Elles sont largement étudiées et observées en stations expérimentales et en exploitations de bovins et de caprins laitiers, et ce depuis de nombreuses années (Mocquot 1980, Rémond et al 1999). Des études conduites dans les exploitations visent à mieux comprendre leurs conditions de mise en œuvre par les éleveurs (Cournut et Dedieu 2005, Rubin et al 2006). Celles menées en stations expérimentales cherchent à appréhender les effets sur la variabilité de la baisse de la production laitière selon les espèces et les stades de lactation, la qualité sanitaire et la com- position du lait (Casu et Labussière 1972, Davis et al 1999, Rémond et Pomiès 2007). L'allongement du temps entre deux traites est plutôt au stade de réflexion dans les exploitations de petits ruminants car les cahiers des charges de nombreuses AOP dans ces filières ne permettent pas leur application. La filière caprine française est déficitaire et doit importer des volumes de lait pour satisfaire la consommation et les besoins des outils industriels. Ces derniers ne souhaitent probablement pas promouvoir des pratiques pouvant induire une diminution des livraisons s'il n'y a pas de compensation. Toutefois, conscients des enjeux liés à l'installation et la reprise d'exploitations d'élevage, et de ce fait, à la pérennité de leur tissu local d'approvisionnement, les industriels, coopératifs ou privés, prennent de plus en plus en compte les souhaits des producteurs de disposer de leviers réduisant les travaux d'astreinte. Les laiteries pourraient tout à fait intégrer cette diversité de pratiques individuelles dans leur stratégie d'approvisionnement tout en s'organisant pour assurer globalement un approvisionnement régulier sur leur bassin de collecte. Certaines AOP (Rocamandour, Roquefort) ont initié des études sur l'impact de la monotraite sur le produit fini (par exemple qualités biochimiques et organoleptiques) afin, si les résultats sont concluants, d'envisager une modification de leur cahier des charges et les faire valider par l'INAO, l'Institut National d'Origine et de la Qualité (Komara et Marnet 2009, Dutot et al 2011). 


\section{2 / Modification des horaires de traite}

La modification des horaires est une autre pratique pour simplifier la traite en élevage avec la suppression d'une traite hebdomadaire (par exemple celle du dimanche soir), la réalisation de trois traites en deux jours ou de deux traites à horaires rapprochés (Pomiès et al 2008). Seulement deux productions sont concernées : les bovins laitiers et les caprins laitiers.

Ces pratiques réduisent occasionnellement la durée du travail d'astreinte ou permettent sa réorganisation au cours de la journée (tableau 5). Par exemple, deux traites rapprochées le matin permettraient aux éleveurs ou aux salariés de se libérer du temps l'après-midi. Les éleveurs aménagent leur travail différemment en se libérant une soirée par semaine (suppression d'une traite hebdomadaire souvent celle du dimanche soir) ou l'après-midi (2 traites à horaires rapprochés). L'impact sur le temps de travail n'est pas aussi important que pour d'autres pratiques simplifiées, mais la souplesse permise pour réorganiser le travail n'est pas négligeable. Sur une lactation complète en élevage bovin laitier, un intervalle de sept heures entre les deux traites semble apporter un bon compromis entre organisation et performances technico-économiques (Pomiès et al 2008). La modification des horaires de traite dans les élevages nécessite cependant d'ajuster certaines pratiques (affourragement et complémentation), sans modifier plus profondément la conduite du troupeau ou des surfaces fourragères. La suppression d'une traite hebdomadaire est relativement fréquent dans les élevages. Selon une enquête du Contrôle Laitier, près de $17 \%$ des éleveurs du Finistère l'appliqueraient (Portier et al 2006). Elle est parfois utilisée en élevage avec d'autres simplifications de la traite (monotraite, fermeture de la salle de traite). La pratique des deux traites à horaires rapprochés est au stade expérimental (Rémond et al 2006). Des études sont réalisées en élevage et en stations expérimentales pour d'une part, évaluer leurs impacts physiologiques et techniques et d'autre part, comprendre leurs conditions de mise en œuvre dans les élevages.

\section{6 / Adoption des pratiques simplifiées dans les élevages}

\section{1 / Nature et importance des pratiques simplifiées selon les productions animales}

En production laitière, un plus grand nombre d'études a été réalisé en ovins qu'en bovins et en caprins du fait de la dimension de la filière et de moyens disponibles en recherche-développement plus importants (humains, structures expérimentales). Dans les exploitations allaitantes (bovin et ovin), les pratiques simplifiées portent sur l'alimentation et la reproduction. En effet l'alimentation constitue le principal poste de travail dans ces exploitations, notamment en période hivernale (Ferris et al 2006). En outre, la filière structure fortement les périodes de vente des animaux et donc les périodes de mises bas, laissant peu de marge de manœuvre aux éleveurs pour les modifier. En production porcine, les pratiques simplifiées portent surtout sur la reproduction (réduction du nombre de périodes de reproduction par an avec des conduites en 4 ou 5 bandes, externalisation de l'élevage des femelles avec les maternités collectives, simplification des procédures de surveillance des mises bas et d'autres événements de reproduction) $\mathrm{du}$ fait du poids de l'atelier naisseur dans le travail des éleveurs (Salaün 2008). Peu de travaux sur les pratiques simplifiées ont été conduits dans la filière avicole, hormis la sélection de souches plus rustiques. Dans cette filière, qui compte des productions très différentes (chair, pondeuses, gavage), les principaux pics de travail sont des activités ponctuelles liées à la mise en place des lots (préparation des bâtiments, litière...) et à la fin des lots (enlèvement des animaux, nettoyage des bâtiments...) (Gallot 2010). L'élevage avicole est souvent un atelier complémentaire, les questions sur le travail sont alors raisonnées sur les autres ateliers, d'autant plus que la souplesse est permise par des cycles de production différables dans le temps pour éviter les pointes de travail et les concurrences avec les autres ateliers agricoles (Gallot 2008). La conduite dans ces élevages est fortement organisée et rationalisée. En élevage avicole et porcin, la maîtrise du travail repose alors essentiellement sur l'automatisation (par exemple de l'alimentation ou du ramassage des volailles) et la délégation (par exemple le nettoyage des bâtiments à des sociétés extérieures) (Gallot 2008, Salaün 2008).

\section{2 / Déterminants de l'adoption de pratiques simplifiées par les éleveurs}

Les pratiques simplifiées ne sont pas adoptées par tous les éleveurs. Les choix de simplifications dépendent de leurs attentes vis-à-vis de leur travail qu'ils expriment de manière différente comme par exemple en élevage laitier : week-ends libérés $(53 \%)$, vacances (41\%), ou disponibilité accrue au quotidien $(29 \%)$, etc. (Guillaumin et al
2005). Trois autres dimensions influencent également les choix des éleveurs. La dimension collective renvoie au fait que les éleveurs ont recours à des pratiques simplifiées différentes selon la main-d'œuvre présente (statut, nombre, âge...) et les réseaux relationnels qu'ils mobilisent (Dufour et Dedieu 2010). Par exemple, l'adoption de la ration complète en élevage bovin laitier est plutôt pratiquée par des collectifs de travail qui comptent plusieurs travailleurs permanents se différenciant par leur nature extra familiale. Ces collectifs de travail sont caractérisés par deux traits principaux : le travail à l'extérieur des conjoints et l'expérience antérieure non agricole d'un de leur membre. Les pratiques de groupage des vêlages et de monotraite des vaches en fin de lactation sont plutôt observées dans des collectifs de travail familiaux avec des parents travaillant bénévolement (Cournut et Hostiou 2010). L'adoption des pratiques simplifiées dépend également des objectifs de production des éleveurs, de leurs capacités d'investissement et de la phase dans laquelle se situe l'exploitation (installation ou fin de carrière). Le contexte économique de production influence également les choix des éleveurs toujours en fonction de leur objectif. Par exemple, en fin de campagne laitière, des éleveurs peuvent passer en monotraite saisonnière pour ne pas dépasser leur droit à produire sans trop pénaliser le potentiel de leur troupeau. Les pratiques simplifiées dépendent aussi d'une dimension subjective que sont les rapports entre l'éleveur et ses animaux, son métier, ses aspirations professionnelles, personnelles et familiales (Fiorelli et al 2010). Ainsi l'adoption de pratiques simplifiées ne peut pas être réfléchie sans prendre en compte ces trois dimensions qui expliquent que toute simplification n'est pas possible ou envisageable pour les éleveurs.

\section{7 / Discussion - Conclusion}

\section{1 / Les pratiques simplifiées, un levier essentiel pour amélio- rer le travail des éleveurs}

Cette synthèse confirme que de nombreuses et diverses pratiques simplifiées sont étudiées dans les élevages herbivores (bovin, ovin, caprin) et granivores (porc, volaille), avec cependant des différences entre les productions. Le levier «pratiques simplifiées» est très étudié dans les élevages d'herbivores pour s'affranchir du travail d'astreinte, gérer les concurrences entre activités ou encore préserver du temps pour les activités personnelles des éleveurs. Dans les élevages de granivores, l'intégration 
forte par l'aval s'accompagne de normes tendues vers la rationalité technique et économique de l'atelier et laisse donc peu de place à la simplification. Les leviers «recomposition de la maind'œuvre» et «équipement-automatisation» sont alors les principaux leviers mobilisés dans ces productions (Gallot 2008, Salaün 2008). Comme le montre cette étude, la notion de simplification recouvre plusieurs sens, car elle varie selon la personnalité de chacun : réduire le temps de travail, diminuer la pénibilité (physique et mentale), modifier la répartition des tâches dans l'année en évitant des moments difficiles à gérer, ou encore sécuriser le fonctionnement du système d'élevage. Cette étude a permis d'identifier deux principaux types de simplification: d'une part, les pratiques simplifiées pour réduire le nombre d'actes techniques et ainsi la durée du travail d'astreinte et d'autre part, les pratiques pour jouer sur d'autres dimensions du travail (charge mentale, répartition des tâches pendant l'année en les étalant ou les concentrant...). L'adoption de pratiques simplifiées dans un élevage pour répondre à une ou plusieurs attentes sur le travail a parfois des répercussions plus globales sur le système et les équilibres recherchés. Cela amène à faire face à des contradictions entre certains objectifs des éleveurs mais ces pratiques ont l'avantage d'être très souvent réversibles. Les résultats de cette synthèse confirment que les pratiques simplifiées sont raisonnées par les éleveurs comme un élément majeur pour obtenir de la marge de manœuvre et de la flexibilité dans l'organisation du travail en élevage (Darnhofer et al 2010). L'éleveur en tant que travailleur et organisateur de son travail peut en effet simplifier la conduite d'élevage pour réduire son travail au quotidien ou à certaines périodes, ou ajuster ses rythmes de travail (Cournut et Dedieu 2005). Si certaines pratiques simplifiées induisent une diminution des performances animales et/ou économiques de l'exploitation, c'est bien la recherche d'un équilibre entre économie, technique et travail qui guide les choix et les décisions des éleveurs. La simplification des pratiques d'élevage ne signifie pas toujours une diminution de la productivité animale ou économique comme le montrent certaines études (Olaizola et al 2008). Les pratiques simplifiées pourraient avoir des incidences sur les performances environnementales mais qui n'ont pas été mises en évidence par les experts enquêtés et la bibliographie consultée. En outre, les compromis et les choix se construisent également en mobilisant d'autres leviers que sont la recomposition de la main-d'œuvre et/ou l'investissement dans des équipements et du matériel (Dedieu et Servière 2001).

\section{2 / Limites de l'étude}

Cette étude n'est pas exhaustive car certaines pratiques pour simplifier le travail au quotidien avec le troupeau (réduction de l'âge au sevrage des porcelets, adaptation des pratiques d'hygiène à la traite, fréquence de nettoyage des bâtiments, soins préventifs anticipés pour limiter les interventions curatives subies...) n'ont pas été prises en compte car elles ont été peu évoquées par les experts et par la bibliographie consultée. De même, nous n'avons pas pris en compte les pratiques qui viseraient à simplifier le travail de saison avec les surfaces fourragères (techniques sans labour par exemple) ou avec le troupeau, car ce n'était pas l'objet de l'étude qui était centrée sur le travail au quotidien avec les animaux. Ces simplifications sont cependant un enjeu dans les élevages pour gérer les périodes de pointe et les concurrences entre ateliers. En outre, nous n'avons pas abordé l'efficacité de ces pratiques, ainsi que les conditions favorisant leur mise en œuvre. Ces éléments sont repris dans des «fiches témoignages», en particulier dans les élevages d'herbivores (Moreau et al 2004, Bellet 2007). Notre étude s'est basée sur le recueil d'expériences auprès des experts du développement et des chercheurs, mais sans prendre en compte directement les points de vue des éleveurs, hormis à travers les nombreuses fiches témoignages. Ils mériteraient d'être plus clairement explicités en particulier pour mieux appréhender les conditions d'adoption des pratiques ainsi que leur insertion dans les élevages. En effet, ces simplifications sont considérées comme des pratiques particulières c'est-à-dire innovantes dans les élevages. Les éleveurs ont donc un rôle à jouer pour concevoir des innovations qui tiennent compte de leurs attentes de travail, afin d'améliorer la durabilité des élevages.

\section{3 / Quelles pratiques simpli- fiées pour les systèmes d'élevage de demain?}

Nos résultats ont également montré que les pratiques ne sont pas spécifiques d'une production. En effet, nombre de ces pratiques simplifiées sont utilisées dans les différentes productions d'herbivores et parfois de granivores. Les évolutions futures des systèmes d'élevage pourraient d'ailleurs inciter les filières à adapter certaines pratiques développées dans d'autres productions. Par exemple, l'externalisation de la reproduction, couramment utilisée en élevage porcin avec les maternités collectives, se développe actuellement en élevage laitier avec l'achat des génisses de renouvellement (Rotz et al 2005). Des interrogations se posent sur le rôle que jouera le levier de la simplification des pratiques pour les élevages de demain, c'est-àdire qui soient compétitifs et rentables, respectueux de l'environnement et répondant aux souhaits des éleveurs : quelles simplifications des conduites d'élevage sont à concevoir pour répondre aux évolutions futures des systèmes d'élevage? Est-ce que l'innovation existera encore ? En effet, l'adaptation des conduites d'élevage est toujours délicate à aborder par son côté déstabilisant pour les éleveurs du fait de l'obligation du maintien des performances techniques, de la sensibilité de la trésorerie au cours de la période de transition, et des faibles marges de manœuvre. Les éleveurs préfèrent alors souvent recourir aux solutions touchant la mécanisation et l'automatisation, plus maîtrisables et prévisibles (étude de projet chiffrée). L'agrandissement des structures de production (Kristensen et al 2005) pourrait d'ailleurs orienter vers un plus grand recours à l'automatisation et la délégation du travail. L'exemple des filières granivores va d'ailleurs dans ce sens (Roguet et al 2011). Dans les pays du Nord de l'Europe (Pays-bas, Danemark), avec l'agrandissement des troupeaux laitiers, le robot de traite rencontre un succès important au contraire de la monotraite (Bijl et al 2007). Des questions de recherche-développement se posent quant à l'utilisation du pâturage dans ces élevages : circulation des troupeaux au pâturage, accessibilité entre les surfaces pâturées et le robot de traite (Ketelaar de Lauwere et al 2000). Pour faciliter l'utilisation du robot de traite, l'automatisation se développe avec la circulation des vaches au robot régulée par des barrières anti-retour et des portes de tri automatiques. En élevage ovin laitier, l'automatisation (identification électronique et portillon électronique en salle de traite pour séparer les brebis) pourrait faire évoluer la distribution de l'alimentation en passant d'une ration unique à une distribution individuelle selon le niveau de production des femelles. La simplification pourrait devenir un ensemble d'ajustements, au plus près du travail réel, des protocoles d'exécution de tâches prédéfinies par la filière et des enjeux environnementaux. Les investissements, de plus en plus conséquents dans les élevages, trouveront peut-être leur limite dans des contextes économiques fluctuants. Justement, le modèle danois, basé sur une mécanisation très élevée et qui demande de forts capitaux, est confronté à de grosses difficultés (Perrot et al 2011). Des exploitations offrant de la souplesse et de la flexibilité pour s'ajuster à leur environnement ont de l'avenir. La souplesse offerte par ces pratiques de simplifications souvent réversibles s'avèrerait alors un levier d'adaptation primordial pour assurer la 
flexibilité des systèmes d'élevage (Dedieu et Ingrand 2010).

\section{Remerciements}

Les auteurs remercient sincèrement tous les experts et relecteurs des insti- tuts techniques (Institut de l'Elevage, IFIP, ITAVI), de l'INRA, des stations expérimentales du Mourier (CIIRPO) et de Jalogny ainsi que des Chambres d'Agriculture (03-22-35-42-43-58-6372-89) qui ont répondu à l'enquête et ainsi participé à la formalisation de ce travail.

\section{Références}

Armstrong D.P., Ho C., 2007. Economic impact of switching to once-a-day milking on a dairy farm in northern Victoria. AFBM Journal, 6, 55-62.

Audist M.J., O’Brien G.O., Cole D. Macmillan K.L., Grainger C., 2007. Effects of varying lactation length on milk production capacity of cows in pasture-based dairying systems. J. Dairy Sci., 90, 3234-3241.

Barthez A., 1986. Du labeur paysan au métier d'agriculteur : l'élaboration statistique en agriculture. Cah. Econ. Sociol. Rurales, 3, 45-72.

Bartolomeu D., 2005. Les temps de travaux en élevage, points clés et facteurs de variation. Techni Porc, 28, 1-10.

Bastien D., Chaigneau F., Molle J., 2009. Réduction du temps de travail d'alimentation en bâtiment des bovins viande : distribution des rations trois fois par semaine. Renc. Rech. Rum., 16, 183-186.

Bellet V., 2007. Gagner du temps en élevage ovin en Poitou Charentes ...des expériences positives mises en place par les éleveurs. Etude du Département Actions Régionales (DAR). Institut de l'Elevage, Paris, France, 117p.

Bellet V., Coquil X., Gomas A.L., 2010. Synthèse des ateliers : Aborder le travail en élevage : un changement de posture nécessaire qui ouvre des voies de recherche et de développement. 3 eme Rencontres Nationales Travail en Elevage, $6 \mathrm{p}$.

Benoit M., Laignel G., 2009. Performances techniques et économiques en élevage biologique d'ovins viande: observations en réseaux d'élevage et fermes expérimentales. In : Numéro spécial, Elevage bio. Perez J.M. (Ed). INRA Prod. Anim., 22, 197-206.

Bijl R., Kooistra S.R., Hogeveen H., 2007. The profitability of automatic milking on dutch dairy farms. J. Dairy Sci., 90, 239-248.

Bossis N., 2006. Stratégies de reproduction, 5 cas concrets en région Centre. Etude du Département Actions Régionales (DAR), Institut de l'Elevage, Paris, France, 28p.

Broccard V., Portier B., Le Meur D., 2005. Groupage des vêlages en systèmes économes premiers enseignements pratiques. Renc. Rech. Rum., 12, 169.

Broccard V., Portier B., Le Meur D., Porhiel J.Y., Lopez C., 2007. Traire les vaches une fois par jour ou grouper les vêlages : deux techniques pour réduire le travail d'astreinte en élevage. Renc. Rech. Rum., 14, 393-386.

Brunschwig P., Plouzin D., 2007. Simplification de l'allaitement et du sevrage des veaux laitiers. Renc. Rech. Rum., 14, 267.

Caillaud D., 2006. Rations sèches : plus de lait, moins de travail, mais à quel prix ?
Etude du Département Actions Régionales (DAR), Institut de 1'Elevage, Paris, France, $11 \mathrm{p}$

Caramelle-Holtz E., Chauvat S., Ethève F., Kentzel M., Moreau J.C., Morin E., 2004. Le travail dans les exploitations d'élevage d'Aquitaine. Compte-rendu. Institut de l'Elevage, Paris, France, 71p.

Caramelle-Holtz E., Bossis N., Guinamard C. Crémoux R, 2008. Produire du lait au bon moment pour répondre aux attentes des entreprises et des éleveurs. Institut de l'Elevage, Paris, France, $45 \mathrm{p}$

Casu S., Labussière J., 1972. Premiers résultats concernant la suppression d'une ou plusieurs traites par semaine chez la brebis Sarde. Ann. Zootech., 21, 223-232.

Chatellier V., Pflimlin A., Perrot C., 2008. La production laitière dans les régions de l'arc Atlantique européen. INRA Prod. Anim., 21, 427-440.

Chauvat S., Seegers J., N'Guyen The B., Clément B., 2003. Le travail d'astreinte en élevage bovin laitier. Institut de l'Elevage, Paris, France, 50p

Clark D.A., Phyn C.V.C., Tong M.J., Collis S.J, Dalley D.E., 2006. A systems comparison of once- versus twice-daily milking of pastured dairy cows. J. Dairy Sci., 89, 1854-1862.

Cournut S., Dedieu B., 2005. Simplification des conduites d'élevage en bovins laitiers. Cah. Agric., 14, 541-547.

Cournut S., Hostiou N., 2010 Adaptation des systèmes bovin laitier pour réduire la contrainte travail. Une étude en Ségala. Cah. Agric., 19, 348-353.

Cutullic E., Delaby L., Gallard Y., Disenhaus C., 2010. L'effet de la stratégie d'alimentation sur la reproduction des vaches laitières varie selon la race et les différentes phases du cycle de reproduction. Renc. Rech. Rum., 17, 149-152.

Darnhofer I., Bellon S., Dedieu B., Milestad R., 2010. Adaptiveness to enhance the sustainability of farming systems. A review. Agron. Sustain. Dev., 30, 545-555.

Davis S.R., Farr V.C., Stelwagen K., 1999 Regulation of yield loss and milk composition during once-daily milking : a review. Livest. Prod. Sci., 59, 77-94.

Dedieu B., Servière G., 2001. Organisation du travail et fonctionnement des systèmes d'élevage. Renc. Rech. Rum., 8, 245-250.

Dedieu B., Ingrand S., 2010. Incertitude et adaptation : cadres théoriques et application l'analyse de la dynamique des systèmes d'élevage. In : Robustesse, rusticité, flexibilité, plasticité, résilience,... les nouveaux critères de qualité des animaux et des systèmes d'élevage.
Sauvant D., Perez J.M., (Eds). Dossier, INRA Prod. Anim., 23, 81-90.

Delaby L., Faverdin P., Disenhaus C., Peyraud J.L., 2009. Effect of different feeding strategies on lactation performance of Holstein and Normande dairy cows. Animal, 3, 891905 .

Dufour A., Dedieu B., 2010. Rapports au temps de travail et modes d'organisation en élevage laitier. Cah. Agric., 19, 377-382.

Dutot S., Durand G., Gaudru M.L., Martin B., Pomiès D., Hulin S., Marnet P.G., 2011. La monotraite chez les éleveurs caprins fromagers : conséquences dans le cadre de l'AOP Rocamadour. Renc. Rech. Rum., 18.

Ferris C.P., Frost J., Binnie R.C., Patterson D.C., 2006. Dairy cows performance and labour inputs associated with two silage feeding systems. Grass Forage Sci., 61, 304-314.

Ferris C.P., Frost J.P., Mayne C.S., McCoy M.A., Kilpatrick D.J., 2008. A comparison of the direct and residual response of dairy cows to once or twice-daily milking, in late lactation. Livest. Sci., 114, 305-314.

Fiorelli C., Porcher J., Dedieu B., 2010. Un cadre d'analyse des compromis adoptés par les éleveurs pour organiser leur travail. Cah. Agric., 19, 383-390

Gallot S., 2008. Le travail en aviculture. Synthèse bibliographique. Itavi, Paris, France, 23p.

Gallot S., 2010. Référentiel travail en élevages avicoles. Synthèse de 24 Bilans Travail. Document de la collection Partenaires RMT travail. Itavi, Paris, France, p20.

Glesson D., O’Brien B., O’Donovan., 2998. The labour input associated with calf care on Irish dairy farms. Livest. Sci., 116, 82-89.

Grannec M.L., 2010. Référentiel Travail en élevages porcins. Synthèse de 23 Bilans Travail réalisés en Bretagne et Pays de la Loire. Document de la collection Partenaires RMT travail. Itavi, Paris, France, 20p.

Guillaumin A., Kling-Eveillard F., Moreau J.C., Marty M., 2005. Résultats d'enquêtes en Aquitaine. Quand les éleveurs laitiers parlent de leurs conditions de travail. Travaux et Innovations, 115, 30-34.

Huchon J.C., Goulard L., Désarménien D. Sabatté N., Gaboriau L., Rubin B., 2006. A la recherche de solutions pour améliorer le travail en élevage laitier : groupage des vêlages. Fiche $n^{\circ} 9,4 \mathrm{p}$

Kentzel M., 2007. Améliorer les conditions de travail dans les systèmes bovins viande d'Aquitaine. Les solutions de réduction du travail en élevage. Etude du Département Actions Régionales. Institut de l'Elevage, Paris, France, $12 \mathrm{p}$. 
Ketelaar-de Lauwere C.C., Ipema A.H., Lokhorst C., Metz J.H.M., Noordhuizen J.P.T.M., Schouten W.G.P., Smits A.C., 2000. Effect of sward height and distance between pasture and barn on cows' visits to an automatic milking system and other behaviour. Livest. Prod. Sci., 65, 131-142.

Komara M., Marnet P.G., 2009. Conduite en monotraite chez la chèvre alpine : application dès la mise bas ou après une à trois semaines de traite biquotidienne ou de conduite mixte monotraite/tétée ? Renc. Rech. Rum., 16, 179182.

Komara M., Boutinaud M., Ben Chedly H., Guinard-Flament J., Marnet P.G., 2009. Oncedaily milking effects in high-yielding alpine dairy goats. J. Dairy Sci., 92, 5447-5455.

Kristensen T., Soegaard K., Kristensen I.S., 2005. Management of grasslands in intensive dairy livestock farming. Livest. Prod. Sci., 96, 61-73.

Larousse, 2009. Dictionnaire en ligne. http://www.larousse.fr/dictionnaires/francais/.

Losq G., Brunschwig P., 2009. Rations sèches pour vaches laitières : résultats d'enquête en élevages. Renc. Rech. Rum., 16, 194.

Losq G., Porhiel J.Y., Portier B., Seite Y., Trou G., 2009. Des solutions pour alléger le travail d'astreinte dans les systèmes laitiers. Renc. Rech. Rum., 16, 198.

Madelrieux S., Dedieu B., 2008. Qualification and assessment of work organization in livestock farms. Animal, 2, 453-446.

Martel G., Dourmad J.Y., Dedieu B., 2008. Do labour productivity and preferences about work load distribution affect reproduction management and performance in pig farms? Livest. Sci., 118, 96-117.

Mocquot J.C., 1980. La réduction du nombre de traites : mythe ou réalité ? Chèvre, 25-31.

Moreau J.C., Seegers J., Kling-Eveillard F., 2004. Répondre à la préoccupation travail des éleveurs: compte-rendu du programme national 2003 financé par l'Onilait. 1ère partie: 1er recueil d'expériences sur les solutions possibles en exploitation. Institut de l'Elevage, Paris, France, 37p.
Morin E., 2003. Etaler sa production laitière sur le rayon de Roquefort : problèmes rencontrés, résultats possibles. Institut de l'Elevage, Paris, France, 27p.

Morin E., de Boissieu C., 2011. Produire du lait de brebis, réfléchir à sa période de traite. Eléments techniques, repères économiques et conditions de réussite. Institut de l'Elevage. http://www.inst-elevage.asso.fr/spip.php?article20548

Olaizola A.M., Chertouh T., Manrique E., 2008. Adoption of a new feeding technology in Mediterranean sheep farming systems: Implications and economic evaluation. Small Rum. Res., 79, 137-145.

Perrot C., Mottet A, You G, 2011, Les modèles laitiers européens du nord de l'UE à l'épreuve de la volatilité. Renc. Rech. Rum., 18, 317-320.

Pomiès D., Marnet P.G., Cournut S., Barillet J., Guinard-Flament B., Rémond B., 2008. Les conduites de traite simplifiées en élevage laitier : vers la levée de l'astreinte biquotidienne. INRA Prod. Anim., 21, 59-70.

Porhiel J.Y., 2008. Allonger la lactation pour un vêlage tous les 18 mois. Premiers résultats à la station de Trévarez. Cap Elevage, 21, 12-14.

Portier B., Chénais F., Broccard V., 2006. Traire une fois par jour toute l'année : un revenu équivalent à certaines conditions. Cap Elevage, 6, 10-13

Rattin S., 2006. Le travail s'intensifie dans les exploitations professionnelles toujours agrandies. Agreste Cah., 4, 3-12.

Rémond B., Pomiès D., 2007. Once-daily milking of Holstein cows for one-week decreases milk yield by twenty-five percent without any carry-over effect. Livest. Sci., 110, 192-195.

Rémond B., Coulon J.B., Nicloux M., Levieux D., 1999. Effect of temporary oncedaily milking in early lactation on milk production and nutritional status of dairy cows. Ann. Zootech., 48, 341-352.

Rémond B, Pomiès D, Julien C., Pradel P, 2006. Effets de faibles écarts de temps entre les deux traites de la journée sur la quantité de lait produite et sa composition, chez la vache laitière. Renc. Rech. Rum., 13, 365-368.

Riedel J.L., Casasús I., Bernués A., 2007. Sheep farming intensification and utilization of natural resources in a Mediterranean pastoral agro-ecosystem. Livest. Sci., 111, 153-163.

Roguet C., Massabie P., Ramonet Y., Grannec M.L., Rieu M., 2011. Quels modèles d'élevage d'avenir pour la production porcine française ? Innov. Agron., 17, 109-124.

Rotz C.A., Zartman D.L., Crandall K.L., 2005. Economic and environmental feasibility of a perennial cow dairy farm. J. Dairy Sci., 88, 3009-3019.

Rubin B., Huchon J.C., Sabatte N., Désarménien D., Gaboriau L., Goulard L., 2006. Robot de traite, monotraite, embauche d'un salarié : impacts sur les systèmes fourragers des exploitations en Pays-de-la-Loire. Fourrages, 185, 63-72.

Salaün Y., 2008. Le travail en production porcine : synthèse bibliographique à partir des études conduites par l'Institut du porc et ses partenaires. Ifip, Paris, France, $4 \mathrm{p}$.

Seegers J., Moreau J.C., Beguin E., Guillaumin A., Frappat B., 2006. Attentes des éleveurs laitiers vis-à-vis de leurs conditions de travail et évolution de leurs systèmes d'exploitation. Fourrages, 185, 3-16

Seite Y., Brocard V., Portier B., Realland B., Losq G., 2009. Affourrager les vaches laitières une fois par semaine pour réduire le temps d'alimentation. Renc. Rech. Rum., 16, 195.

Sidot G., 2006. L'efficacité du travail et la qualité de vie dans les élevages laitiers en France et en Europe. Fourrages, 185, 17-24.

Sorensen A., Donald Muir D., Knight C.H., 2008. Extended lactation in dairy cows: effects of milking frequency, calving season and nutrition on lactation persistency and milk quality. J. Dairy Res., 75, 90-97.

Trou G., Gaudillière N., Disenhaus C., Jouanne D., Le Guenic M., Portier B., Roger P. 2009. Motivations, pratiques et résultats de seize éleveurs bretons se ménageant cinq à six mois sans vêlage. Renc. Rech. Rum, 16, 175178.

\section{Résumé}

Les questions sur le travail sont depuis de nombreuses années au cour des préoccupations des éleveurs et du monde professionnel. La simplification des pratiques d'élevage est une des voies de résolution des problèmes de travail des éleveurs. Cet article cherche d'une part, à mettre en évidence les pratiques simplifiées actuellement en discussion dans les productions herbivores et granivores et d'autre part, à préciser la notion de «simplification». Cette notion est subjective, elle varie selon la personnalité de chacun et recouvre plusieurs sens (réduire le temps de travail, diminuer la pénibilité, s'organiser différemment...). Une enquête auprès d'experts des différentes filières animales et une recherche bibliographique ont été réalisées pour caractériser les pratiques simplifiées (nature, incidences sur le travail, filières concernées...). Différentes composantes du système d'élevage, du fait des fortes charges en travail, peuvent être simplifiées : l'alimentation, la reproduction et la traite. Les pratiques simplifiées sont très étudiées dans les élevages d'herbivores pour s'affranchir du travail d'astreinte, gérer les concurrences entre activités, ou encore préserver du temps pour les activités personnelles et familiales. Pour les exploitations de granivores, l'intégration forte par l'aval s'accompagne de normes tendues vers la rationalité technique et économique optimale de l'atelier et laisse peu de place à la simplification. Les leviers «recomposition de la main-d'œuvre» et «équipement-automatisation» sont alors les principaux leviers mobilisés dans ces élevages. Des interrogations se posent sur le rôle que jouera le levier de la simplification des pratiques pour les élevages de demain, c'est-à-dire qui soient compétitifs et rentables, respectueux de l'environnement et répondant aux objectifs des éleveurs. La souplesse offerte par les pratiques simplifiées, souvent réversibles, pourrait s'avérer être un levier primordial pour assurer la flexibilité et les capacités d'adaptation des élevages dans un contexte de plus en plus incertain. 


\begin{abstract}
Simplification of livestock management: an analysis of simplified practices developed in herbivore and grain-fed production systems

For many years, the preoccupations of livestock farmers and the professional world have been focused on questions about work. Simplifying livestock management is one way to resolve the work problems of livestock farmers. Our article has sought to emphasize the simplified practices presently under discussion in herbivores and grain-fed production systems as well as to define the notion of "simplification". This notion is subjective; it varies with each and every personality and covers several meanings (reducing the working time, making the work less arduous, organizing it in a different way...). A survey was carried out with experts from different animal productions along with bibliographical research, in order to characterize simplified practices (their nature, their effects on work, the supply chains concerned...). Various features of the livestock system can be simplified because of their heavy workloads: feeding, reproduction and milking. Simplified practices are very closely studied in herbivore livestock farming to enable the farmers to have some respite from routine work, to manage the competition between activities, or even reserve time for personal and family activities. For farms with grain-fed stock, powerful vertical integration is accompanied by standards tending towards optimal technical and economic rationality of the unit and leaves little room for simplification. "Restructuring the workforce" and "automation equipment" are the main levers mobilized in such livestock farms. Questions are raised as to the role that will be played by the "simplification of practices" lever for the livestock farms of tomorrow, i.e. farms that are competitive and profitable, environmentally-friendly and meeting the expectations of the farmers. The flexibility offered by simplified practices that are often reversible will prove to be an essential lever in ensuring that livestock farms have the necessary capacities to adapt to an increasingly uncertain context.
\end{abstract}

HOSTIOU N., FAGON J., 2012. Simplification des conduites d'élevage : analyse transversale des pratiques mises en œuvre dans les filières herbivores et granivores. In : Numéro spécial, Travail en élevage. Hostiou N., Dedieu B., Baumont R. (Eds). INRA Prod. Anim., 25, 127-140. 